\title{
Erratum
}

\section{Preferred Shapes and Modes of Internal Motion in a Five-Boson System}

\author{
C.-G. Bao ${ }^{1}$, J.-Z. Pan ${ }^{2}$, and T. K. Lim $^{3}$
}

1 Deparment of Physics, Zhong-Shan University, Guangzhou, People's Republic of China

2 Institute of High-Energy Physics, Beijing, People's Republic of China

3 Department of Physics and Atmospheric Science, Drexel University, Philadelphia, PA 19104, USA

[Few-Body Systems 9, 25 (1990)]

(i) On page 33, line 10, ref. [2] should read ref. [3].

(ii) On pages 34 and 35, the contents of Subsection 4.7 should be:

We have compared the ground-state energies of the three systems for $V_{0}=$ $300[1,4]$ and have found that the "binding energy per pair of particles", i.e. $E_{0} /(n(n-1) / 2)$, are $-28.16,-28.3$, and $-25.0 \mathrm{MeV}$ as we go from the three- to the five-body systems. This seems to suggest that the tetrahedron is the most favourable to binding. The energy gap between the ground and first excited states for the systems are $30.98,26.2$, and $63.3 \mathrm{MeV}$. Thus the ground state of the five-body system is the most stable against monopole excitation. 